\title{
One Class of Splitting Iterative Schemes
}

\author{
Čiegis and V. Pakalnytė \\ Vilnius Gediminas Technical University \\ Saulètekio al. 11, 2054, Vilnius, Lithuania \\ rc@fm.vtu.lt
}

\begin{abstract}
This paper deals with the stability analysis of a new class of iterative methods for elliptic problems. These schemes are based on a general splitting method, which decomposes a multidimensional parabolic problem into a system of one dimensional implicit problems. We use a spectral stability analysis and investigate the convergence order of two iterative schemes. Finally, some results of numerical experiments are presented.
\end{abstract}

\section{Introduction}

Iterative methods for solving discrete elliptic problems can be viewed as finite difference schemes for non stationary parabolic problems. The most important difference is that for elliptic problems we select the time step parameter according the requirements of the convergence to the stationary solution and can ignore the approximation error. For splitting methods for elliptic problems we refer to $[4,6]$. In particular, [5,6] involve an alternating direction method, [4] presents factorization schemes. The convergence rate can be increased if optimal nonstationary parameters are used for the definition of each iteration, see e.g. [6].

Recently the multicomponent versions of alternating direction method were proposed in [1]. These schemes are also used for solving multidimensional elliptic problems [2]. While in the previous papers the stability of these splitting schemes was investigated by the energy method, we will use the spectral stability analysis. For symmetric problems it gives necessary and sufficient convergence conditions and enables us to find optimal values of iterative parameters. Such analysis was used also in [3]

The content of this paper is organized as follows. In Section 2 we formulate the multicomponent iterative scheme. The convergence of $2 \mathrm{D}$ scheme is investigated in Section 3. In section 4 we investigate the stability of 3D iterative scheme, the analysis is done using numerical experiments. Finally, in Section 5 we study the convergence of the $p$-dimensional multicomponent iterative scheme and prove the energy stability estimates. In Section 6 , the Seidel type scheme is formulated and investigated. 


\section{Multicomponent iterative scheme}

Let the strictly elliptic problem be given by

$$
\begin{array}{r}
-\sum_{i=1}^{p} \frac{\partial^{2} u}{\partial x_{i}^{2}}=f(x), \quad x \in Q, \\
u(x)=0 \quad x \in \partial Q,
\end{array}
$$

where $Q=(0,1)^{p}$. We introduce a uniform grid in $Q$ and approximate the elliptic problem by the following finite difference scheme

$$
\sum_{\alpha=1}^{p} A_{\alpha} y=f
$$

here $A_{\alpha}$ denotes the approximation of a differential operator using standard central difference formula.

Let $($,$) and \|\cdot\|$ denote the inner product and the $L_{2}$ norm of discrete functions, respectively. Generally, we introduce the following assumptions:

$$
\begin{aligned}
A_{\alpha}^{*} & =A_{\alpha}, \\
0<m_{\alpha}\|y\| & \leq\left(A_{\alpha} y, y\right) \leq M_{\alpha}\|y\| .
\end{aligned}
$$

It is well known that for the Laplace operator these assumptions are satisfied with

$$
8 \leq m_{\alpha}, \quad M_{\alpha} \leq \frac{4}{h^{2}}
$$

where $h$ is the spatial step size.

In order to solve the system of linear equations (1) we define $p$ unknown functions $y_{\alpha}, \alpha=1,2, \ldots, p$. Then the multicomponent alternating direction (MAD) scheme is given as (see, [1]):

$$
\begin{aligned}
& \frac{\stackrel{s+1}{y}_{\alpha}-\stackrel{s}{y}}{\tau}+p A_{\alpha}\left(\stackrel{s+1}{y}_{\alpha}-\stackrel{s}{y}_{\alpha}\right)+\sum_{\beta=1}^{p} A_{\beta} \stackrel{s}{y}_{\beta}=f, \quad \alpha=1,2, \ldots, p \\
& \stackrel{s}{\tilde{y}}=\frac{1}{p} \sum_{\alpha=1}^{p} \stackrel{s}{y}_{\alpha}
\end{aligned}
$$

where $\stackrel{s}{y}_{\alpha}$ is the $s$-th iteration of $y_{\alpha}$. We note that all equations (3) can be solved in parallel.

We will investigate the convergence of the MAD scheme by using the following error norms:

$$
\|\stackrel{s}{e}\|=\|\stackrel{s}{\tilde{y}}-y\|, \quad\|\stackrel{s}{r}\|=\left\|\sum_{\alpha=1}^{p} A_{\alpha} \stackrel{s}{y}_{\alpha}-f\right\| .
$$




\section{Spectral stability analysis of $2 \mathrm{D}$ iterative scheme}

In this section we consider a two-dimensional iterative scheme (1)

$$
\frac{\stackrel{s+1}{y_{\alpha}-\tilde{y}}}{\tau}+2 A_{\alpha}\left(\stackrel{s+1}{y}_{\alpha}-\stackrel{s}{y}_{\alpha}\right)+\sum_{\beta=1}^{2} A_{\beta} \stackrel{s}{y}_{\beta}=f, \quad \alpha=1,2
$$

Let denote the error functions

$$
\stackrel{s}{e_{\alpha}}=\stackrel{s}{y}_{\alpha}-y, \quad \alpha=1,2, \ldots, p, \quad \stackrel{s}{\tilde{e}}=\stackrel{s}{\tilde{y}}-y .
$$

Then the error functions satisfy the following MAD scheme:

$$
\begin{aligned}
& \frac{\stackrel{s+1}{e_{\alpha}}-\stackrel{s}{\tilde{e}}}{\tau}+2 A_{\alpha}\left(\stackrel{s+1}{e_{\alpha}}-\stackrel{s}{e_{\alpha}}\right)+\sum_{\beta=1}^{2} A_{\beta} \stackrel{s}{e_{\beta}}=0, \quad \alpha=1,2, \\
& \stackrel{s}{\tilde{e}}=\frac{1}{2} \sum_{\alpha=1}^{2} \stackrel{s}{e_{\alpha}},
\end{aligned}
$$

To apply the discrete von Neumann stability criteria to problem (5), we let

$$
\stackrel{s}{e}_{\alpha}=\sum_{j=1}^{N-1} \sum_{k=1}^{N-1} \stackrel{s}{d} d_{\alpha, j k} \sin \left(j \pi x_{1}\right) \sin \left(k \pi x_{2}\right), \quad \alpha=1,2,
$$

where $N$ is the number of grid points in one-dimensional grid. It is well known, that $\sin \left(j \pi x_{\alpha}\right)$ are eigenvectors of the operator $A_{\alpha}$, i.e.:

$$
\begin{array}{r}
A_{\alpha} \sin \left(j \pi x_{\alpha}\right)=\lambda_{j} \sin \left(j \pi x_{\alpha}\right), \\
8 \leq \lambda_{1} \leq \lambda_{2} \leq \cdots \leq \lambda_{N-1} \leq \frac{4}{h^{2}} .
\end{array}
$$

If we replace $\stackrel{s+1}{e_{\alpha}}$ and $\stackrel{s}{e_{\alpha}}$ in (5) by expressions of the form given by equation (6) and use (7), we get the following matrix equations

$$
\stackrel{s+1}{\mathbf{d}_{j k}}=Q_{2} \stackrel{s}{\mathbf{d}_{j k}},
$$

where $\mathbf{d}_{j k}$ is the column vector of spectral coefficients

$$
\mathbf{d}_{j k}=\left(\begin{array}{c}
d_{1, j k} \\
d_{2, j k}
\end{array}\right)
$$

and $Q_{2}$ is the stability matrix of MAD scheme

$$
Q_{2}=\left(\begin{array}{cc}
\frac{0.5+\tau \lambda_{j}}{1+2 \tau \lambda_{j}} & \frac{0.5-\tau \lambda_{k}}{1+2 \tau \lambda_{j}} \\
\frac{0.5-\tau \lambda_{j}}{1+2 \tau \lambda_{k}} & \frac{0.5+\tau \lambda_{k}}{1+2 \tau \lambda_{k}}
\end{array}\right)
$$


Now we consider the necessary conditions for the stability of iterative MAD scheme (4). Since $Q_{2}$ is not symmetric matrix, the discrete von Neumann stability criteria can not prove that they are also sufficient for stability of MAD scheme.

Theorem 1. All eigenvalues of stability matrix $Q_{2}$ satisfy inequalities

$$
\left|q_{j k}\right|<1, \quad 1 \leq j, k \leq N-1
$$

unconditionally for any values of parameters $\tau$ and $h$.

Proof. Using simple computations we get that eigenvalues $q$ of the amplification matrix $Q_{2}$ satisfy the quadratic equation

$$
q^{2}-q+\frac{\tau\left(\lambda_{j}+\lambda_{k}\right)}{\left(1+2 \tau \lambda_{j}\right)\left(1+2 \tau \lambda_{k}\right)}=0 .
$$

Then the eigenvalues of $Q_{2}$ are

$$
q_{1,2}=\frac{1}{2}\left(1 \pm \sqrt{\frac{\left(1-2 \tau \lambda_{j}\right)\left(1-2 \tau \lambda_{k}\right)}{\left(1+2 \tau \lambda_{j}\right)\left(1+2 \tau \lambda_{k}\right)}}\right)
$$

and they are obviously both less than 1 . The theorem is proved.

The convergence order of MDA iterative scheme depends on the parameter $\tau$. We will use quasi - optimal parameter $\tau_{0}$ which solves the minimization problem

$$
w\left(\tau_{0}\right)=\min _{\tau} \max _{\lambda_{1} \leq \lambda \leq \lambda_{N-1}}\left|\frac{1-2 \tau \lambda}{1+2 \tau \lambda}\right| .
$$

Since the function

$$
g(a)=\frac{1-a}{1+a}
$$

is strictly decreasing, we find $\tau_{0}$ from the equation

$$
\frac{1-2 \tau \lambda_{1}}{1+2 \tau \lambda_{1}}=\frac{2 \tau \lambda_{N-1}-1}{1+2 \tau \lambda_{N-1}} .
$$

After simple computations we get

$$
\tau_{0}=\frac{1}{2 \sqrt{\lambda_{1} \lambda_{N-1}}} \approx \frac{h}{4 \pi}
$$

and all eigenvalues of the amplification matrix $Q_{2}$ satisfy the inequality

$$
\left|q_{j k}\left(\tau_{0}\right)\right| \leq \frac{1}{1+\sqrt{\lambda_{1} / \lambda_{N-1}}} \approx 1-\frac{\pi}{2} h .
$$

These estimates are similar to convergence estimates obtained for the standard iterative scheme of alternating directions. 


\section{Spectral stability analysis of 3D iterative scheme}

As it was stated in section 3, the von Neumann stability criteria gives only sufficient stability conditions of MAD iterative scheme. In this section we will use the spectral stability analysis for 3D iterative scheme (3). But instead of finding eigenvalues of an amplification matrix $Q_{3}$ we use direct numerical experiments and find the optimal value of the parameter $\tau$. Such methodology gives us a possibility to investigate the convergence order of MAD scheme in the usual $l_{2}$ norm.

Let consider the model problem

$$
\sum_{\alpha=1}^{3} A_{\alpha} y=\left(\lambda_{j}+\lambda_{k}+\lambda_{l}\right) \sin \left(j \pi x_{1}\right) \sin \left(k \pi x_{2}\right) \sin \left(l \pi x_{3}\right)
$$

which has the exact solution

$$
y=\sin \left(j \pi x_{1}\right) \sin \left(k \pi x_{2}\right) \sin \left(l \pi x_{3}\right) .
$$

Then the solution of 3D MAD scheme can be represented as

$$
\stackrel{s}{y}_{\alpha}=\stackrel{s}{d}_{\alpha} \sin \left(j \pi x_{1}\right) \sin \left(k \pi x_{2}\right) \sin \left(l \pi x_{3}\right), \quad \alpha=1,2,3,
$$

where $d_{\alpha}$ can be computed explicitly

$$
\stackrel{s+1}{d}{ }_{\alpha}=\frac{1}{1+3 \tau \lambda_{\alpha}}\left(\sum_{\beta=1}^{3}\left(\frac{s}{d_{\beta}}-\tau \lambda_{\beta}\left(\stackrel{s}{d}_{\beta}-1\right)\right)+3 \tau \lambda_{\alpha} \stackrel{s}{d}\right), \quad \alpha=1,2,3 .
$$

Then the error of the sth iteration $\stackrel{s}{\tilde{d}}$ is estimated by the following formula

$$
\stackrel{s}{e}=\left|\frac{\stackrel{s}{d_{1}+d_{2}+\stackrel{s}{d}}-1}{3}-1\right|
$$

Let $S\left(\tau, \lambda_{j}, \lambda_{k}, \lambda_{l}\right)$ be the number of iterations required to achieve the accuracy $\stackrel{s}{e} \leq \varepsilon$ for given eigenvalues $\lambda_{\alpha}$. Then we investigate the whole interval $[m, M]$, which characterize the stiffness of the problem and compute

$$
S(\tau)=\max _{m \leq \lambda_{\alpha} \leq M} S\left(\tau, \lambda_{j}, \lambda_{k}, \lambda_{l}\right)
$$

This problem is solved approximately by computing $S\left(\tau, \lambda_{j}, \lambda_{k}, \lambda_{l}\right)$ for $(K+1)^{3}$ combinations of eigenvalues $\lambda_{\alpha}=m+i(M-m) / K$.

First, we investigated the dependence of the number of iterations $S(\tau)$ on the parameter $\tau$. It was proved that there exists the optimal value $\tau_{0}$, such that

$$
S\left(\tau_{0}\right) \leq S(\tau)
$$


Table 1. The number of iterations as a function of $\tau$

\begin{tabular}{ccc}
\hline$\varepsilon$ & $\tau$ & $S(\tau)$ \\
\hline 0.0001 & 0.0015 & 210 \\
0.0001 & 0.0020 & 159 \\
0.0001 & 0.0022 & 145 \\
0.0001 & 0.0023 & 149 \\
0.0001 & 0.0025 & 162 \\
\hline
\end{tabular}

and this value satisfies the following condition

$$
\max _{m \leq \lambda_{\alpha} \leq M} S\left(\tau_{0}, \lambda_{j}, \lambda_{k}, \lambda_{l}\right)=S\left(\tau_{0}, m, m, m\right)
$$

In Table 1 we present numbers of iterations $S(\tau)$ for different values of $\tau$. These experiments were done with $m=10, M=4000$.

The optimal value of the parameter $\tau$ depends slightly on $\varepsilon$. In Table 2 we present optimal numbers of iterations $S\left(\tau_{0}\right)$ for different values of $\varepsilon$.

Table 2. The optimal value of $\tau$ as a function of $\varepsilon$

\begin{tabular}{lcc}
\hline \multicolumn{1}{c}{$\varepsilon$} & $\tau_{0}$ & $S\left(\tau_{0}\right)$ \\
\hline 0.001 & 0.00233 & 103 \\
0.0001 & 0.00221 & 144 \\
0.00001 & 0.00212 & 187 \\
\hline
\end{tabular}

Finally, we investigated the dependence of the convergence rate of the MAD iterative scheme on $m$ and $M$. In Table 3 we present optimal values of $\tau$ and numbers of iterations $S\left(\tau_{0}\right)$ for different spectral intervals. We used $\varepsilon=10^{-4}$ in these experiments.

It follows from results presented in Table 3 that

$$
\tau_{0}=\frac{c}{\sqrt{m M}}, \quad S\left(\tau_{0}\right)=O\left(\sqrt{\frac{M}{m}}\right) .
$$

The above conclusion agrees well with results of section 3 . 
Table 3. The optimal value of $\tau$ as a function of $m$ and $M$

\begin{tabular}{cccc}
\hline$m$ & $M$ & $\tau_{0}$ & $S\left(\tau_{0}\right)$ \\
\hline 10 & 4000 & 0.00221 & 144 \\
10 & 16000 & 0.00110 & 284 \\
10 & 64000 & 0.00055 & 563 \\
& & & \\
10 & 16000 & 0.00110 & 284 \\
40 & 16000 & 0.00055 & 145 \\
90 & 16000 & 0.00037 & 98 \\
\hline
\end{tabular}

\section{Error estimates for $p$-dimensional $M A D$ scheme}

In this section we consider $p$-dimensional iterative scheme (3). Let us introduce the following notation:

$$
\begin{aligned}
& \stackrel{s}{y}_{\alpha t}=\frac{\stackrel{s+1}{y_{\alpha}-\stackrel{s}{y}_{\alpha}}}{\tau}, \quad \stackrel{s}{y}_{t}=\frac{\stackrel{s+1}{\tilde{y}}-\tilde{s}}{\tau}, \quad \stackrel{s}{v}(\alpha, \beta)=\stackrel{s}{y}_{\alpha}-\stackrel{s}{y}_{\beta} \\
& \|\stackrel{s}{v}\|_{3}^{2}=\sum_{\alpha, \beta=1, \alpha>\beta}^{p}\|\stackrel{s}{v}(\alpha, \beta)\|^{2}, \quad Q_{p}(\stackrel{s}{y})=\|\stackrel{s}{r}\|^{2}+\frac{1}{p^{2} \tau^{2}}\|\stackrel{s}{v}\|_{3}^{2} .
\end{aligned}
$$

In the following theorem we estimate the convergence rate of $M A D$ iterative scheme.

Theorem 2. Iterative scheme (3) produces a sequence converging unconditionally to the solution of problem (2) and the convergence rate is estimated as

$$
Q_{p}(\stackrel{s+1}{y}) \leq \frac{1}{q} Q_{p}(\stackrel{s}{y}), \quad q=\min \left(1+m p \tau, 1+\frac{1}{2 M p \tau}\right)
$$

where $m$ and $M$ are the spectral estimates of the operator $A$ :

$$
m=\min _{1 \leq \alpha \leq p} m_{\alpha}, \quad M=\max _{1 \leq \alpha \leq p} M_{\alpha}
$$

Proof. Multiplying both sides of (3) by $\stackrel{s}{y}_{\alpha t}$ and adding all equalities we get

$$
\sum_{\alpha=1}^{p}\left(\frac{\stackrel{s+1}{y}_{\alpha}-\tilde{s}}{\tau}, \stackrel{s}{y}{ }_{\alpha t}\right)+p \tau \sum_{\alpha=1}^{p}\left(A_{\alpha} \stackrel{s}{y}_{\alpha t}, \stackrel{s}{y_{\alpha t}}\right)+p\left(\sum_{\beta=1}^{p} A_{\beta} \stackrel{s}{y_{\beta}}-f, \stackrel{s}{\tilde{y}_{t}}\right)=0
$$


The first term of (13) can be rewritten as

$$
\begin{aligned}
& I_{1}=\frac{1}{p \tau} \sum_{\alpha=1}^{p} \sum_{\beta=1}^{p}\left(\stackrel{s+1}{y}_{\alpha}-\stackrel{s+1}{y}_{\beta}, \stackrel{s}{y_{\alpha t}}\right)+\frac{1}{p \tau} \sum_{\alpha=1}^{p} \sum_{\beta=1}^{p}\left(\stackrel{s+1}{y}_{\beta}-\stackrel{s}{y}_{\beta}, \stackrel{s}{y}_{\alpha t}\right) \\
& =p\left\|\stackrel{s}{\tilde{y}_{t}}\right\|^{2}+\frac{1}{p \tau} \sum_{\alpha, \beta=1, \alpha>\beta}^{p}\left({ }^{s+1}(\alpha, \beta), s_{t}^{(\alpha, \beta)}\right) \\
& =p\left\|\stackrel{s}{\tilde{y}_{t}}\right\|^{2}+\frac{1}{2 p}\left\|\stackrel{s}{v}_{t}\right\|_{3}^{2}+\frac{1}{2 p \tau^{2}}\left(\|\stackrel{s+1}{v}\|_{3}^{2}-\|\stackrel{s}{v}\|_{3}^{2}\right) .
\end{aligned}
$$

By adding equations (3) we get that

$$
\stackrel{s}{y}_{t}=-\left(\sum_{\alpha=1}^{p} A_{\alpha} \stackrel{s+1}{y}_{\alpha}-f\right)
$$

hence using the third term of (13) we can prove that

$$
p\left\|\stackrel{s}{\tilde{y}_{t}}\right\|^{2}+p\left(\sum_{\beta=1}^{p} A_{\beta} \stackrel{s}{y_{\beta}}-f, \stackrel{s}{\tilde{y}_{t}}\right) \geq \frac{p}{2}\left(\|\stackrel{s+1}{r}\|^{2}-\|\stackrel{s}{r}\|^{2}\right) .
$$

The second term of (13) is estimated similarly. Thus the convergence rate estimate (12) follows trivially. The theorem is proved.

As a corollary of Theorem 2 we can find the optimal value of parameter $\tau_{0}=1 / p \sqrt{2 m M}$, which is obtained from the equation

$$
1+p m \tau=1+\frac{1}{2 M p \tau} .
$$

\section{Seidel-type iterative scheme}

In this section we investigate the convergence rate of Seidel-type iterative scheme:

$$
\begin{aligned}
& \frac{\stackrel{s+1}{y_{\alpha}}-\stackrel{s}{y}_{\alpha}^{*}}{\tau}+\sum_{\beta=1}^{\alpha} A_{\beta} \stackrel{s+1}{y_{\beta}}+\sum_{\beta=\alpha+1}^{p} A_{\beta} \stackrel{s}{y_{\beta}}=f, \quad \alpha=1,2, \ldots, p, \\
& \stackrel{s}{y}_{1}^{*}=\stackrel{s}{y_{1}}, \quad \stackrel{s}{y}_{\alpha}^{*}=0.5\left(\stackrel{s}{y}_{\alpha}+\stackrel{s}{y}_{\alpha-1}\right) \text {. }
\end{aligned}
$$

\subsection{Spectral stability analysis of $2 \mathrm{D}$ scheme}

To apply the discrete von Neumann stability criteria to problem (16), we write the global error as a series:

$$
\stackrel{s}{e_{\alpha}}=\sum_{j=1}^{N-1} \sum_{k=1}^{N-1} \stackrel{s}{d} d_{\alpha, j k} \sin \left(j \pi x_{1}\right) \sin \left(k \pi x_{2}\right), \quad \alpha=1,2,
$$


Substituting this expansion into (16), we obtain the equation for coefficients

$$
\stackrel{s+1}{\mathbf{d}} j k_{j k} Q_{2} \stackrel{s}{\mathbf{d}_{j k}} .
$$

where $\mathbf{d}_{j k}$ is the column vector of spectral coefficients and $Q_{2}$ is the stability matrix of scheme (16)

$$
Q_{2}=\left(\begin{array}{cc}
\frac{1}{1+\tau \lambda_{j}} & \frac{-\tau \lambda_{k}}{1+\tau \lambda_{j}} \\
\frac{0.5\left(1-\tau \lambda_{j}\right)}{\left(1+\tau \lambda_{j}\right)\left(1+\tau \lambda_{k}\right)} & \frac{0.5+\tau \lambda_{j}+\tau^{2} \lambda_{j} \lambda_{k}}{\left(1+\tau \lambda_{j}\right)\left(1+\tau \lambda_{k}\right)}
\end{array}\right) .
$$

Now we consider the necessary conditions for the stability of scheme (16). The eigenvalues of the amplification matrix $Q_{2}$ satisfy the quadratic equation

$$
q^{2}-\left(1+\frac{0.5\left(1-\tau \lambda_{j}\right)}{\left(1+\tau \lambda_{j}\right)\left(1+\tau \lambda_{k}\right)}\right) q+\frac{0.5}{1+\tau \lambda_{j}}=0 .
$$

Theorem 3. All eigenvalues of stability matrix $Q_{2}$ satisfy inequalities

$$
\left|q_{j k}\right|<1, \quad 1 \leq j, k \leq N-1
$$

unconditionally for any values of parameters $\tau$ and $h$.

Proof. Application of the Hurwitz criterion gives that $\left|q_{j k}\right| \leq 1$ is satisfied if and only if

$$
\frac{0.5}{1+\tau \lambda_{j}}<1, \quad\left|1+\frac{0.5\left(1-\tau \lambda_{j}\right)}{\left(1+\tau \lambda_{j}\right)\left(1+\tau \lambda_{k}\right)}\right|<1+\frac{0.5}{1+\tau \lambda_{j}} .
$$

Simple computations prove that both inequalities are satisfied unconditionally. The theorem is proved.

\subsection{Spectral stability analysis of $3 \mathrm{D}$ iterative scheme}

Let consider the model problem (10). The solution of 3D scheme (16) can be represented as

$$
\stackrel{s}{y}_{\alpha}=\stackrel{s}{d} \alpha \sin \left(j \pi x_{1}\right) \sin \left(k \pi x_{2}\right) \sin \left(l \pi x_{3}\right), \quad \alpha=1,2,3,
$$

where $d_{\alpha}, \alpha=1,2,3$, are computed explicitly

$$
\stackrel{s+1}{d} \alpha=\frac{1}{1+\tau \lambda_{\alpha}}\left(\frac{\stackrel{s}{d}+\stackrel{s}{d} d_{\alpha-1}}{2}-\sum_{\beta=1}^{\alpha-1} \tau \lambda_{\beta} \stackrel{s+1}{d} \beta-\sum_{\beta=\alpha+1}^{3} \tau \lambda_{\beta} \stackrel{s}{d} \beta+\sum_{\beta=1}^{3} \tau \lambda_{\beta}\right) .
$$

We estimate the error of the sth iteration $\stackrel{s}{d}$ 作 by the following formula

$$
\stackrel{s}{e}=\max _{1 \leq \alpha \leq 3}\left|\begin{array}{l}
s \\
d \alpha
\end{array}\right| \text {. }
$$


Table 4. The optimal value of $\tau$ as a function of $m$ and $M$

\begin{tabular}{cccc}
\hline$m$ & $M$ & $\tau_{0}$ & $S\left(\tau_{0}\right)$ \\
\hline 10 & 4000 & 0.00173 & 176 \\
10 & 16000 & 0.00089 & 346 \\
10 & 64000 & 0.00045 & 681 \\
& & & \\
10 & 16000 & 0.00089 & 346 \\
40 & 16000 & 0.00042 & 177 \\
90 & 16000 & 0.00028 & 125 \\
\hline
\end{tabular}

We investigated numerically the dependence of the convergence rate of the iterative scheme (16) on $m$ and $M$. In Table 4 we present optimal values of $\tau$ and numbers of iterations $S\left(\tau_{0}\right)$ for different spectral intervals. We used $\varepsilon=10^{-4}$ in these experiments.

It follows from results presented in Table 4 that

$$
\tau_{0}=\frac{c}{\sqrt{m M}}, \quad S\left(\tau_{0}\right)=O\left(\sqrt{\frac{M}{m}}\right) .
$$

The convergence rate of the Seidel type scheme (16) is the same as of scheme (3).

\section{References}

1. Abrashin, V.N.: On the stability of multicomponent additive direction method. Differentsialnyje uravnenyja. 35 (1999) 212-224 (in Russian).

2. Abrashin, V.N., Zhadaeva, N.G.: On the convergence rate of economical iterative methods for stationary problems of mathematical physics. Differentsialnyje uravnenyja. 36 (2000) 1422-1432 (in Russian).

3. Aleinikova, T., Čiegis, R.: Investigation of new iterative methods for solving multidimensional elliptic equations. Differentsialnyje uravnenyja. 29 (1993) 1124-1129 (in Russian).

4. Marchuk, G.I. Splitting methods. Nauka, Moscow, 1988. (in Russian).

5. Peaceman, D., Rachford, H.: The numerical solution of parabolic and elliptic differential equations. SIAM, 3 (1955).

6. Samarskii, A.A., Nikolajev, E.S. Methods for solving difference equations. Nauka, Moscow, 1978.(in Russian). 